\title{
Re-examination of Structure Optimization of Off-Lattice Protein AB Models by Conformational Space Annealing
}

\author{
JINWOO LEE, ${ }^{1,2}$ KEEHYOUNG JOO, ${ }^{2}$ SEUNG-YEON KIM, ${ }^{3}$ JOOYOUNG LEE ${ }^{2}$ \\ ${ }^{1}$ Department of Mathematics, Kwangwoon University, 26 Kwangoon Street, Nowon-Gu, \\ Seoul 139-701, Korea \\ ${ }^{2}$ School of Computational Sciences, Korea Institute for Advanced Study, \\ 207-43 Cheongryangri-dong, Dongdaemun-gu, Seoul 130-722, Korea \\ ${ }^{3}$ School of Liberal Arts and Sciences, Chungju National University, Chungju 380-702, Korea \\ Received 1 September 2007; Revised 4 February 2008; Accepted 6 March 2008 \\ DOI 10.1002/jcc.20995 \\ Published online 12 May 2008 in Wiley InterScience (www.interscience.wiley.com).
}

\begin{abstract}
The global structural optimization is carried out for off-lattice protein AB models in two and three dimensions by conformational space annealing. The models consist of hydrophobic and hydrophilic monomers in Fibonacci sequences. To accelerate the convergence, we have introduced a shift operator in the internal coordinate system, and effectively reduced the search space by forming a quotient space. With this, we significantly improve our previous results on $\mathrm{AB}$ models, and provide new low energy conformations. This work provides insights on exploring complicated energy landscapes by exploiting the advantages and limitations of CSA.
\end{abstract}

(C) 2008 Wiley Periodicals, Inc. J Comput Chem 29: 2479-2484, 2008

Key words: global optimization; structure optimization; protein model; energy landscape

\section{Introduction}

Protein structure prediction is one of the challenging problems of this century in computational sciences. Based on Anfinsen's thermodynamic hypothesis, ${ }^{1}$ computational framework for protein structure prediction is well established, defining an appropriate energy function and its optimization. Given an energy function, finding the global minimum of it in the protein folding is a hard combinatorial optimization problem. Recently the importance of optimization in all stages of protein structure prediction using bioinformatics is also investigated. ${ }^{2}$

Simplified protein models such as $\mathrm{AB}$ models ${ }^{3}$ are devised to study qualitative properties of protein folding. Off-lattice protein $\mathrm{AB}$ models consist of hydrophobic monomers labeled by $\mathrm{A}$ and hydrophilic ones by $\mathrm{B}$. Interactions between these monomers are composed of a Lennard-Jones type potential term, a bending energy term, and torsional energy contributions.

Although the coarse-grained models of proteins are quite simplified, their free-energy landscapes are quite rugged and finding their global minima is a nontrivial task. Although putative groundstate conformations have been reported, they are continually being updated, ${ }^{4-9}$ and thus it is not clear if they correspond to true ground-state conformations.

In ref. 6, a global optimization method called conformational space annealing $(\mathrm{CSA})^{10-12}$ is applied to find ground-state con- formations of protein $\mathrm{AB}$ models. At that time, optimization by CSA was terminated when a new structure was obtained with lower energy than reported in the literature. Thus systematic and rigorous optimization was not performed. In addition, there was a confusion in signs of energy terms of model2 (see Off-Lattice AB Models section below).

In this article, we study systematically ground-state properties of protein $\mathrm{AB}$ models by using CSA. A distinguished point from ref. 6 is that a "shift operator" is introduced to accelerate the convergence. It effectively reduces the search space, and new low energy conformations are reported for four separate cases.

\section{Off-Lattice AB Models}

Two effective off-lattice AB models are investigated with Fibonacci sequences. The Fibonacci sequence is defined recursively by

$$
\Lambda_{0}=A, \quad \Lambda_{1}=B, \Lambda_{i}=\Lambda_{i-2} * \Lambda_{i-1},
$$

Correspondence to: J. Lee; e-mail: jlee@kias.re.kr Contract/grant sponsor: Kwangwoon University 


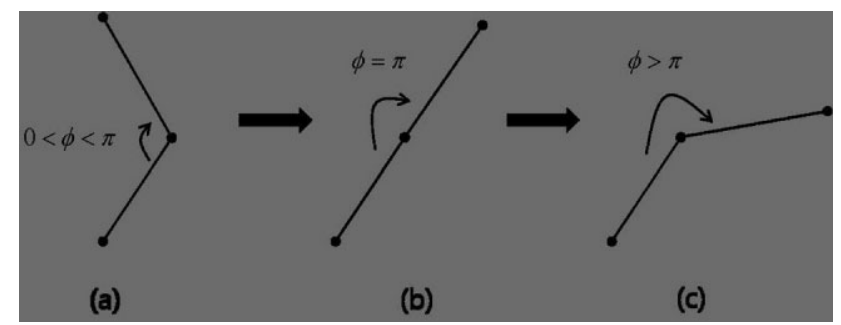

Figure 1. A series of structural changes during minimization in terms of a bond angle is shown. A positive bond angle of (a) goes through a value $\phi=\pi$ at (b), and becomes $\phi>\pi$ (or equivalently $\phi<0$ ).

where the asterisk denotes the concatenation operator. We study the cases of $6 \leq i \leq 9$. The sequences of chains are denoted as $S_{13}, S_{21}, S_{34}$, and $S_{55}$ for $i=6,7,8$, and 9, respectively, where subscripts indicate chain-sizes.

The first model investigated in this work is the original AB model. It was proposed in ref. 3 with the energy function

$$
E_{1}=\sum_{i=1}^{N-2} \frac{1}{4}\left(1-\cos \theta_{i, i+1}\right)+4 \sum_{i=1}^{N-2} \sum_{j=i+2}^{N}\left[\frac{1}{r_{i j}^{12}}-\frac{C_{1}\left(\sigma_{i}, \sigma_{j}\right)}{r_{i j}^{6}}\right],
$$

where $\theta_{i, j}$ is the angle between the $i$ th and $j$ th bond vectors (c.f. it is not a bond angle, but $\pi-$ bond angle) and $r_{i j}$ is the distance between monomers $i$ and $j$. The constant $C_{1}\left(\sigma_{i}, \sigma_{j}\right)$ is $+1,+1 / 2$, and $-1 / 2$ for $\mathrm{AA}, \mathrm{BB}$, and $\mathrm{AB}$ pairs, respectively, thus introducing strong attraction between AA pairs, weak attraction for BB pairs, and weak repulsion between $\mathrm{AB}$ pairs. The bending energy term induces chains to expand, and thus partially competes with the van der Waals energy term. All bond lengths are fixed to unity. We call this model "model1" throughout this article, and simulations are performed in two and three dimensions.

The second model denoted here as model 2 was introduced in ref. 13. The energy is given by

$$
\begin{aligned}
E_{1}=\sum_{i=1}^{N-2} \cos \theta_{i, i+1} & -\frac{1}{2} \sum_{i=1}^{N-3} \cos \theta_{i, i+2} \\
& +4 \sum_{i=1}^{N-2} \sum_{j=i+2}^{N} C_{2}\left(\sigma_{i}, \sigma_{j}\right) \times\left(\frac{1}{r_{i j}^{12}}-\frac{1}{r_{i j}^{6}}\right),
\end{aligned}
$$

where $C_{2}\left(\sigma_{i}, \sigma_{j}\right)$ is +1 for $\mathrm{AA}$ pairs and $+1 / 2$ for $\mathrm{BB}$ and $\mathrm{AB}$ pairs. Therefore all nonbonded interactions are attractive with emphasis on stronger AA interactions. The first and second terms prefer chains to zigzag. Thus all terms compete with each other. All bond lengths are fixed to unity. Since the second term was introduced in ref. 13 to mimic a torsional energy term, the model 2 is investigated only in three dimensions.

\section{Methods}

\section{Shift Operator}

In this work, conformations are represented by the internal coordinate $I$, and there exist trivial symmetries in $I$. First, after performing local energy minimization of a conformation, a certain bond angle $\phi_{i}$ (initially $\left.0 \leq \phi_{i} \leq \pi\right)$ can be of a negative value $\left(-\pi \leq \phi_{i} \leq \pi\right)$. Since any conformation can be represented with bond angles in the range of 0 and $\pi$, in this case, we have another representation of the conformation with negative "bond angles." Performing restrained local minimization is not a remedy to this problem. Since the energy function is continuous in terms of the internal coordinate, the range of "bond angles" should not be restricted to reach a local minimum of a given conformation.

Suppose two bonds are in a shape shown in Figure 1a with a positive bond angle before minimization, and it undergoes the structural change through Figures $1 \mathrm{~b}$ and $1 \mathrm{c}$ in a continuous manner (to make the situation simple, we assume that all the other angles of the rest of the molecule are fixed during minimization). If the local minimization is performed in terms of cartesian coordinates, no special operator is required. However, using the internal coordinate system, the final shape (Fig. 1c) is described with a negative (or $\phi>\pi$ ) bond angle. To represent the final structure using the bond angle in the range of $[0, \pi]$, we reassign the bond angle of Figure 1 when the shape crosses the flat shape $(\phi=\pi)$ of Figure $1 \mathrm{~b}$ so that the bond angle remains in the range of $[0, \pi]$. This was carried out by introducing the shift operator.

Second, changing signs of all dihedral angles $\left(-\pi \leq \psi_{i} \leq \pi\right)$ of a conformation in a simultaneous fashion yields the mirror image with identical energy value.

The idea of forming reduced space is to "glue together" if two points in $I$ represent the same conformation or are mirror images from each other. Mathematically speaking, we define an equivalence relation $\sim$ and obtain a reduced space by forming a quotient space $I / \sim$.

For $p, q \in I$, let $p=\left(\phi_{1}^{p}, \psi_{1}^{p}, \phi_{2}^{p}, \psi_{2}^{p}, \cdots, \psi_{n-2}^{p}, \phi_{n-1}^{p}\right)$, and $q=\left(\phi_{1}^{q}, \psi_{1}^{q}, \phi_{2}^{q}, \psi_{2}^{q}, \cdots, \psi_{n-2}^{q}, \phi_{n-1}^{q}\right)$, where $\phi_{i}^{(\cdot)}$ are "bond angles" (which can be negative values, $-\pi \leq \phi_{i}^{(\cdot)} \leq \pi$ ), and $-\pi \leq \psi_{i}^{(\cdot)} \leq$ $\pi$ are dihedral angles. The number of monomers is denoted as $n$. The index system is that the adjacent dihedral angles of $\phi_{i}^{(\cdot)}$ become $\psi_{i-1}^{(\cdot)}$ and $\psi_{i}^{(\cdot)}$. The equivalent relation $\sim$ is defined by the following: $p \sim q$ if (1) $\left[\phi_{i}^{p}=-\phi_{i}^{q}, \psi_{i-1}^{p}=\psi_{i-1}^{q}+\pi\right.$, and $\left.\psi_{i}^{p}=\psi_{i}^{q}+\pi\right]$ for some $i$, and $\left[\phi^{p}=\phi^{q}\right.$ and $\left.\psi^{p}=\psi^{q}\right]$ for the rest of the angles, or (2) $\left[\phi_{i}^{p}=\phi_{i}^{q}\right.$, and $\left.\psi_{i}^{p}=-\psi_{i}^{q}\right]$ for all $i$.

The condition (1) comes from the fact that if a bond angle of a conformation is of a negative value, one can represent the identical conformation by converting the bond angle to a positive value and flipping its two adjacent dihedral angles. As a result, all bond angles can be defined between 0 and $\pi$. The condition (2) corresponds to gluing mirror images into one.

By the definition of (1), $2^{n-1}$ points in $I$ are reduced to a single point in $I / \sim$, and by (2) 2 points in $I$ becomes a single point in $I / \sim$. Thus by the "shift operator," the size of $I / \sim$ is reduced by the factor of $2^{n}$ from the size of $I$.

Although this operator is defined for the specific beads-like protein models, this kind of idea can be applied to any reduced protein 
Table 1. The Lowest Energies of Model1 in the Two-Dimensional AB Model by this Work, in Comparison with Those by nPERMis, ACMC, our Previous Work (Old CSA), and STMD.

\begin{tabular}{lrrrrr}
\hline & nPERMis & ACMC & old CSA & STMD & CSA (this work) \\
\hline$S_{13}$ & -3.2939 & -3.2941 & -3.2941 & -3.2941 & $-3.2941(10 / 10)$ \\
$S_{21}$ & -6.1976 & -6.1979 & -6.1980 & -6.1980 & $-6.1980(10 / 10)$ \\
$S_{34}$ & -8.9749 & -10.8060 & -10.8060 & -10.8060 & $-10.8060(10 / 10)$ \\
$S_{55}$ & -18.5154 & -18.7407 & -18.9110 & -18.9202 & $-\mathbf{- 1 8 . 9 2 9 6}(1 / 10)$ \\
\hline
\end{tabular}

The numbers in the parenthesis in the last column indicates the number of times the lowest energy is found out of ten independent runs. The new lowest energy is shown in bold case. The conformations of the previously reported lowest energy and the current work are shown in Figure 2.

models where virtual bond angles are introduced. ${ }^{11,14}$ The condition (2) is not necessary when dealing with chirally symmetry-broken units such as amino acids of $\mathrm{L}$ and $\mathrm{D}$ types.

Equipped with this shift operator, we have applied CSA to this reduced internal coordinate space $I / \sim$.

\section{Conformational Space Annealing}

The CSA method searches the whole conformational space in its early stages and then narrows the search to smaller regions with low energy as the distance cutoff, $D_{\text {cut }}$, which defines the similarity of two conformations, is reduced. It starts with a preassigned number (50 in this work) of randomly generated and subsequently energyminimized conformations. This pool of conformations is called the bank. At the beginning, the bank is a sparse representation of the conformational space of local minima.

A number of dissimilar conformations (30 in this work) are then selected from the bank, excluding those that have already been used; they are called seeds. Each seed conformation is modified by replacing consecutive parts (from $10 \%$ to $40 \%$ of the chain selected in a random fashion) of the seed by the corresponding parts of a randomly selected conformation from the bank. Each conformation is energy-minimized to give a trial conformation. A total of 30 trial conformations are generated for each seed (hence 900 trial conformations). This corresponds to the most time-consuming part of the algorithm, and consequently it is highly suitable for parallel computation, ${ }^{15}$ since the energy minimizations are independent from each other.

For each trial conformation, $\alpha$, the closest conformation $A$ from the bank (in terms of the root mean square deviation, $d(\alpha, A)$ ) is determined. If $d(\alpha, A) \leq D_{\text {cut }}$ ( $D_{\text {cut }}$ being the current value of cutoff criterion), $\alpha$ is considered similar to $A$; in this case $\alpha$ replaces $A$ in the bank, if it is also lower in energy. If $\alpha$ is not similar to $A$, but its energy is lower than that of the highest-energy conformation in the bank, $B, \alpha$ replaces $B$. If neither of the above conditions holds, $\alpha$ is rejected. The narrowing of the search regions is accomplished by setting $D_{\text {cut }}$ to a large value initially and gradually reducing it as the search progresses.

Special attention is paid to selecting seeds that are far from each other. One round of the procedure is completed when there is no seed to select (i.e., all conformations from the bank have already been used). At the end of each round, all conformations in the bank are designated as unused as seeds. The value of $D_{\text {cut }}$ is also set to the original value. The round is repeated a predetermined number of times ( 2 in this work), after which additional random energyminimized conformations (50 in this work) are added to the bank, and the whole procedure is repeated. In this work, the whole CSA search stops after the complete procedure is finished with 500 bank conformations. More details of CSA can be found elsewhere. ${ }^{10,16,17}$

\section{Results}

In the field of protein structure prediction, carefully prepared knowledge-based energy terms are shown to correlate well with the quality of a model. ${ }^{2}$ Therefore if one can find lower energy conformations, the chances are that the quality of their structures is better. We note that the degrees of freedom of the system $S_{n}$ in three dimensions increases as $2 n$ which corresponds to the number of backbone angles of a protein with $n$ residues.

\section{Model1 in Two Dimensions}

Table 1 shows the results of the lowest energy of model1 in two dimensions, along with the values reported in the literature by nPERMis, ${ }^{4}$ ACMC,${ }^{5}$ our previous work, ${ }^{6}$ and $\mathrm{STMD}^{7}$ for comparison. All methods except nPERMis report identical results for $S_{13}, S_{21}$, and $S_{34}$. We could almost be sure that the results obtained may correspond to the true ground-state energies. However, for $S_{55}$, a lower energy conformation is found in this work. Figure 2 shows our lowest energy conformation along with that of STMD. Although its energy is only slightly lower than that found by STMD, hydrophobic cores of our model are more tightly packed in the sense that the number of hydrophobic residues not packed in the cores is one less than that from STMD. We remark that out of 10 independent runs, the new minimum is reached only once, indicating the energy landscape of $S_{55}$ in two dimensions is quite rugged and thus finding the new conformation is a nontrivial task.
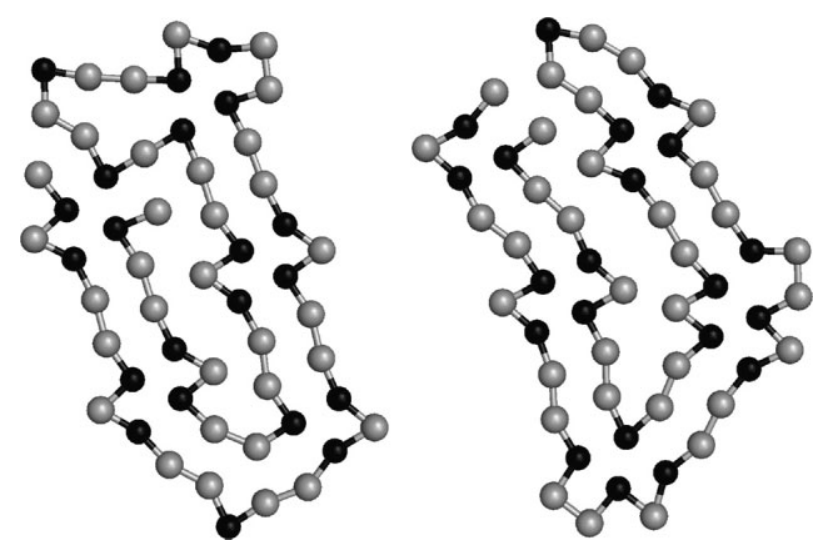

Figure 2. The lowest energy conformation from STMD (left) for $S_{55}$ in two dimensions, and the lowest energy conformation of this study (right) is shown. STMD conformation contains hydrophobic residues of four 4As, one $3 \mathrm{~A}$, and two single As. Our conformation contains one $5 \mathrm{~A}$, three $4 \mathrm{As}$, one $3 \mathrm{~A}$, and one single $\mathrm{A}$. 
Table 2. The Lowest Energies of Model1 in the Three-Dimensional AB Model by this Work, in Comparison with Those by nPERMis, ELP, our Previous Work (Old CSA), and STA.

\begin{tabular}{lrrrrr}
\hline & nPERMis & ELP & old CSA & STA & CSA (this work) \\
\hline$S_{13}$ & -4.9616 & -4.967 & -4.9746 & -4.9746 & $-4.9746(10 / 10)$ \\
$S_{21}$ & -11.5238 & -12.316 & -12.3266 & -12.3266 & $-12.3266(10 / 10)$ \\
$S_{34}$ & -21.5678 & -25.476 & -25.5113 & -25.5113 & $-25.5113(5 / 10)$ \\
$S_{55}$ & -32.8843 & -42.428 & -42.3418 & -42.5781 & $\mathbf{- 4 4 . 7 9 8 3}(2 / 10)$ \\
\hline
\end{tabular}

The numbers in the parenthesis in the last column indicates the number of times the lowest energy is found out of ten independent runs. The new lowest energy is shown in bold case.

\section{Model1 in Three Dimensions}

Table 2 shows the lowest energies of our model 1 in three dimensions, along with the results by nPERMis, ELP,${ }^{8}$ our previous work, and STA. ${ }^{9}$ Recent three methods report identical results for $S_{13}, S_{21}$, and $S_{34}$. However, for $S_{55}$, our result for the lowest-energy conformation shown in Figure 3 is $~ 5 \%$ lower in energy compared with that by STA. In all 10 independent runs, lowest energies found are lower than that of STA. To investigate low-lying regions in the energy landscape, we chose three "stable" (found several times as the lowest energy in 10 runs) conformations and compared their structures. When we denote them as $\mathrm{c} 1, \mathrm{c} 2, \mathrm{c} 3$, their energies are -44.7983 , -44.4212 , and -44.15806 , respectively. TM-scores ${ }^{18}$ between $\mathrm{c} 1$ and the others are about 0.23 meaning $\mathrm{c} 1$ is quite different from $\mathrm{c} 2$ and $\mathrm{c} 3$. However, TM-score between $\mathrm{c} 2$ and $\mathrm{c} 3$ is 0.67 (roughly speaking, $67 \%$ can be superimposable). See the structures in Figure 3. This illustrates a fine structure of low-lying regions in the energy landscape indicating there are two deep basins and one of them has two low-energy meta-stable local minima.

This demonstrates the ability of CSA to cover low-lying energy regions scattered distantly in the energy landscape. This feature of CSA is quite important in the field of protein structure prediction. Low-energy conformations scattered distantly may cover different portions of protein structures, and thus there are more chances to improve the quality of structures by combining the information from diverse low-energy structures. ${ }^{2}$

In the three-dimensional model1, these results strongly suggest that ground-states for $S_{13}, S_{21}$, and $S_{34}$ are found, However, the situation is less clear for the case of $S_{55}$.

\section{Model2 in Three Dimensions}

In the three-dimensional model2, the problem appears to be more difficult. Table 3 shows the lowest energies of our results, along with the results by ACMC, ELP, our previous work (We acknowledge that there were confusions and mistakes on signs in the energy terms in the previous work for model2, and thus the values in the column of old CSA in Table III should be neglected.), and STA. In this case, even the results in $S_{34}$ do not agree from each other. This observation may indirectly indicate that the energy landscapes of model 2 are more rugged than those of model1. This is somewhat expected from the functional form in that there are more competing terms in $E_{2}$. We found the lower energy conformations for $S_{34}$ and $S_{55}$ shown in Figures 4 and 5, respectively. For $S_{34},-98.3571$ is reached only twice out of ten runs. The other 8 runs all reached -98.0757 that is also found as the second lowest energy minimum in the two more successful runs. TM-score between these two conformations is 0.31 indicating they are structurally quite different from each other. This
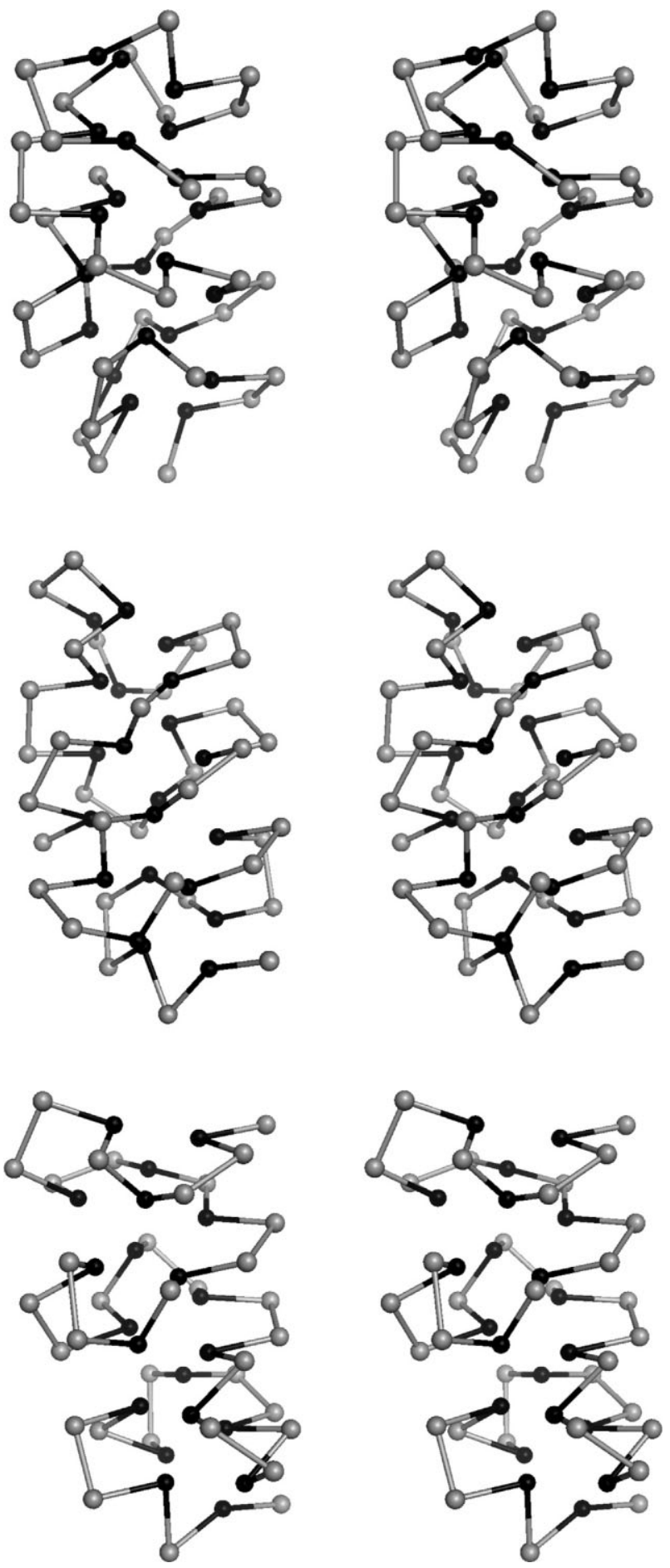

Figure 3. The lowest energy conformation (c1) for $S_{55}$ of model 2 in three dimensions, and two low-energy meta-stable conformations c2 and $\mathrm{c} 3$ are shown in streoview from top to bottom. 
Table 3. The Lowest Energies of Model 2 in the Three-Dimensional AB Model by this Work, in Comparison with Those by ACMC, ELP, our Previous Work (old CSA [18]), and STA.

\begin{tabular}{lcrrrr}
\hline & ACMC & ELP & old CSA & STA & CSA (this work) \\
\hline$S_{13}$ & -26.506 & -26.498 & -26.4714 & -25.5066 & $-26.5066(10 / 10)$ \\
$S_{21}$ & -51.7575 & -52.917 & -52.7865 & -52.9339 & $-52.9339(10 / 10)$ \\
$S_{34}$ & -94.0431 & -92.746 & -97.7321 & -97.6171 & $-\mathbf{9 8 . 3 5 7 1}(2 / 10)$ \\
$S_{55}$ & -154.505 & -172.696 & -173.9803 & -174.5681 & $-\mathbf{1 7 6 . 6 9 1 3}(1 / 10)$ \\
\hline
\end{tabular}

The numbers in the parenthesis in the last column indicates the number of times the lowest energy is found out of ten independent runs. The new lowest energy is shown in bold case.

demonstrates that the energy landscape of $S_{34}$ contains a deep narrow basin and a wider basin. It should be noted that we have found eight distinct conformations whose energy values are all lower than the previous best one by STA. The previous best conformation by STA is also obtained in this study. TM-scores between seven conformations are all less than 0.32 from each other, and the score between two out of eight was 0.5 . This illustrates the CSA's ability to find low energy conformations while keeping diversity between conformations, especially in much rugged energy landscapes.

For $S_{55}$, a conformation with $1.2 \%$ lower energy compared to that by STA is found. Again, in all 10 independent runs, all lowest energies found in this work are lower than that by STA. However, the lowest energies in 10 independent runs are not identical from each other except that the second minimum, -176.4706 , is reached twice. The lowest minimum energy of -176.6913 is reached only once. This illustrates that finding the global minimum of the energy landscape for $S_{55}$ of model 2 is quite difficult even with the state-ofthe-art method, and one can not be sure the lowest energy reported here corresponds to the true global minimum. In Table 4, results of 10 runs for the above four cases are summarized.

\section{Summary and Discussions}

The ground state energies of two off-lattice AB models are investigated by conformational space annealing (CSA). To accelerate the convergence we have defined an equivalent relation $\sim$, and effectively reduced the internal coordinate space $I$ by forming a quotient space $I / \sim$. By applying CSA to $I / \sim$, lower energy conformations are found for $S_{55}$ of model1 in two dimensions, $S_{55}$ of model1 in
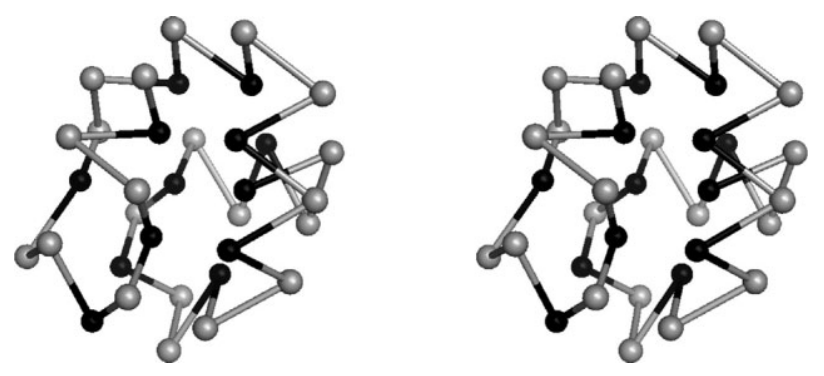

Figure 4. The lowest energy conformation for $S_{34}$ of model 2 in three dimensions is shown in streoview.
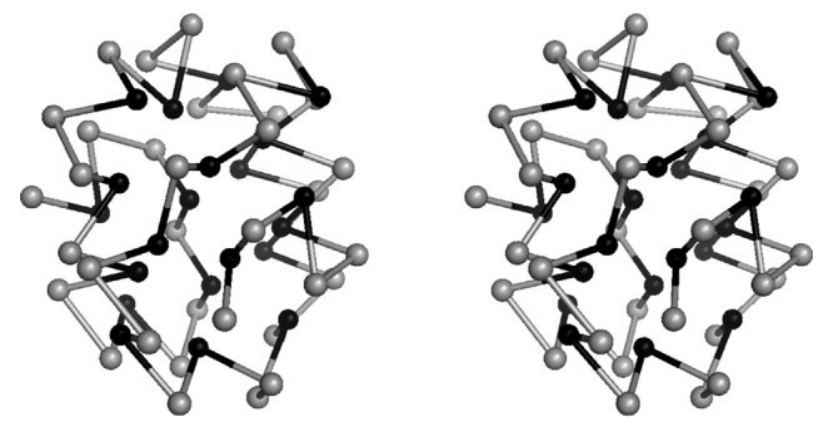

Figure 5. The lowest energy conformation for $S_{55}$ of model 2 in three dimensions is shown in streoview.

three dimensions, $S_{34}$ and $S_{55}$ for model 2 in three dimensions. Fine structures of energy landscapes are also discussed.

For $S_{13}$ and $S_{21}$ of model1 and model2, we could be almost sure that their global minima are found. For $S_{34}$, the ground state conformation of model 1 found by this work is more repeatedly visited than that of model 2 indicating that the energy landscape of model 2 may be more difficult to search than that of model1. It is probably due to one additional competing term in model2. For $S_{55}$, even with the current method, it is difficult to repeatedly find the reported lowest-energy conformations in this work, and thus finding the true global minimum conformations for $S_{55}$ of model1 and model 2 still remains challenging and open to further investigations.

After this work is finished, we have noticed the work of Zhang and $\mathrm{Ma},{ }^{19}$ where new putative ground state conformations are reported for four cases, $2 \mathrm{D} S_{34}, 3 \mathrm{D} S_{55}$ of model1, 3D $S_{34}$ of model2, and $3 \mathrm{D} S_{55}$ of model2. The energies found by Zhang and Ma are lower than those found here in three out of four cases. Here we aim to provide comparative analysis and discussions.

Interestingly, our results for 3D $S_{34}$ of model 2 agree with Zhang and Ma. For this case, previous methods have repeatedly provided new putative global minimum conformations. The agreement from these two separate methods strongly indicates that it could be the true ground-state.

For 2D $S_{55}$ and $3 \mathrm{D} S_{55}$ of model1, Zhang and $\mathrm{Ma}^{19}$ found slightly lower (the energy difference being 0.3274 for $2 \mathrm{D}$, and 0.0782 for $3 \mathrm{D}$, respectively) energy conformations. TM-scores between theirs and the closest one kept in the final bank from CSA was 0.48 for

Table 4. Summary of the Lowest Energies for 10 Independent Runs Found in this Work.

\begin{tabular}{rllll}
\hline Trial & $\begin{array}{l}\text { 2D } S_{55}, \\
\text { Model1 }\end{array}$ & $\begin{array}{l}\text { 3D } S_{55}, \\
\text { Model1 }\end{array}$ & $\begin{array}{l}\text { 3D } S_{34}, \\
\text { Model2 }\end{array}$ & $\begin{array}{c}\text { 3D } S_{55}, \\
\text { Model2 }\end{array}$ \\
\hline 1 & -18.911 & -44.1581 & -98.076 & -176.691 \\
2 & -18.911 & -44.42124 & -98.076 & -175.518 \\
3 & -18.930 & -44.42124 & -98.357 & -176.211 \\
4 & -18.9110 & -44.79829 & -98.076 & -175.721 \\
5 & -18.903 & -44.29656 & -98.076 & -176.471 \\
6 & -18.9110 & -44.15806 & -98.076 & -175.881 \\
7 & -18.9110 & -44.79829 & -98.076 & -175.621 \\
8 & -18.889 & -44.27933 & -98.076 & -175.555 \\
9 & -18.9110 & -44.17801 & -98.357 & -176.471 \\
10 & -18.903 & -44.27094 & -98.076 & -176.067 \\
\hline
\end{tabular}


$2 \mathrm{D}$, and 0.53 for $3 \mathrm{D}$ indicating structural similarity. It implies that CSA may have reached the big basin where the conformations from Zhang and MA lie.

For 3D $S_{55}$ of model2, however, the energy difference is larger (1.4426) and the structural similarity between the two methods is rather low with TM-score of 0.31 .

The failure to obtain lowest energy structures of three protein $\mathrm{AB}$ models using CSA is rather disappointing. However, it is important to understand the reason for the failure and to suggest a possible fix for the problem. One important assumption for the success of $\mathrm{CSA}^{10}$ is that a typical local minimum conformation contains a portion of correct values of internal variables for global minimum solution (combination assumption). In the case of protein structures, most dihedral angles take one of three values denoted as gauche+, gauche- and trans (planar angles take one of the two values, trans and cis). Therefore, a typical local minimum conformation may contain one third of its dihedral angles in agreement with the global minimum conformation (still finding the global minimum remains as a hard combinatorial optimization problem). In CSA, when perturbing a seed conformation, rather than using random variables for random perturbation, randomly chosen locally minimized values from the first bank is used (first bank is the collection of randomly generated and then minimized conformation). We suspect that the current failure is partly from the breakdown of the combination assumption mentioned. Another possibility is that, throughout the CSA procedure, conformations generated are all quite low in energy, especially compared to those by Zhang and Ma. ${ }^{19}$ The fact that the Monte Carlo simulations ${ }^{19-21}$ efficiently generate low and quite high energy conformations, appears to enable one to cover more diverse phase space than sampled by CSA, demonstrating the limitation of CSA especially when the first bank fails to provide appropriate candidate values for the global minimum. To overcome this kind of situation, one needs to introduce new measures to CSA when the combination assumption fails. One possibility could be to combine CSA with generalized Monte Carlo ensemble.

\section{Acknowledgement}

We thank referees for their valuable comments, and KIAS for providing Linux cluster supercomputers.

\section{References}

1. Anfinsen, C. B. Science 1973, 181, 223.

2. Joo, K.; Lee, J.; Lee, S.; Seo, J.; Lee, S. J.; Lee, J. Proteins 2007, 69 Suppl. 8, 83 .

3. Stillinger, F. H.; Head-Gordon, T.; Hirshfeld, C. L. Phys Rev E 1993, $48,1469$.

4. Hsu, H.-P.; Mehra, V.; Nadler, W.; Grassberger, P. J Chem Phys 2003, $118,444$.

5. Liang, F. J Chem Phys 2004, 120, 6756.

6. Kim, S. Y.; Lee, S. B.; Lee, J. Phys Rev E 2005, 72, 011916.

7. Kim, J.; Straub, J. E.; Keyes, T. Phys Rev Lett 2006, 97, 050601.

8. Bachmann, M.; Arkin, H.; Janke, W. Phys Rev E 2005, 71, 031906.

9. Kim, J.; Straub, J. E.; Keyes, T. Phys Rev E 2007, 76, 011913.

10. Lee, J.; Scheraga, H. A.; Rackovsky, S. J Comput Chem 1997, 18, 1222.

11. Lee, J.; Liwo, A.; Scheraga, H. A. Proc Natl Acad Sci USA 1999, 96, 2025.

12. Lee, J.; Lee, I. H.; Lee, J. Phys Rev Lett 2003, 91, 080201.

13. Irbäck, A.; Peterson, C.; Potthast, F.; Sommelius, O. J Chem Phys 1997, 107, 273.

14. Wu, S.; Skolnick, J.; Zhang, Y. BMC Biol 2007, 5, 17.

15. Lee, J.; Pillardy, C.; Czaplewski, J.; Arnautova, Y.; Ripoll, D. R.; Liwo, A.; Gibson, K. D.; Wawak, R. J.; Scheraga, H. A., Comput Phys Commun 2000, 128, 399.

16. Lee, J.; Scheraga, H. A. Int J Quant Chem 1999, 75, 255.

17. Lee, J.; Liwo, A.; Ripoll, D.; Pillardy, J.; Saunders, J. A.; Gibson, K. D.; Scheraga, H. A. Int J Quant Chem 2000, 77, 90.

18. Zhang, Y.; Skolnick, J. Proteins 2004, 57, 702.

19. Zhang, Y. C.; Ma, J. J. Phys Rev E 2007, 76, 036708.

20. Berg, B. A.; Neuhaus, T. Phys Rev Lett 1992, 68, 9.

21. Lee, Y. J. Phys Rev Lett 1993, 71, 211. 